\title{
High-speed simultaneous multiscale photoacoustic microscopy
}

Mohesh Moothanchery

Renzhe Bi

Jin Young Kim

Ghayathri Balasundaram

Chulhong Kim

Malini Olivo 


\title{
High-speed simultaneous multiscale photoacoustic microscopy
}

\author{
Mohesh Moothanchery, ${ }^{a}$ Renzhe Bi, ${ }^{a}$ Jin Young Kim, ${ }^{b}$ Ghayathri Balasundaram, ${ }^{a}$ Chulhong Kim, ${ }^{b, *}$ and \\ Malini Olivo ${ }^{\mathrm{a}, \star}$ \\ ${ }^{a}$ Singapore Bioimaging Consortium, Agency for Science Technology and Research, Singapore \\ ${ }^{b}$ Pohang University of Science and Technology, Department of Creative IT Engineering, Pohang, Republic of Korea
}

\begin{abstract}
Photoacoustic microscopy (PAM) is a fast-growing biomedical imaging technique that provides highresolution in vivo imaging beyond the optical diffusion limit. Depending on the scalable lateral resolution and achievable penetration depth, PAM can be classified into optical resolution PAM (OR-PAM) and acoustic resolution PAM (AR-PAM). The use of a microelectromechanical systems (MEMS) scanner has improved OR-PAM imaging speed significantly and is highly beneficial in the development of miniaturized handheld devices. The shallow penetration depth of OR-PAM limits the use of such devices for a wide range of clinical applications. We report the use of a high-speed MEMS scanner for both OR-PAM and AR-PAM. A high-speed, wide-area scanning integrated OR-AR-PAM system combining MEMS scanner and raster mechanical movement was developed. A lateral resolution of $5 \mu \mathrm{m}$ and penetration depth $\sim 0.9-\mathrm{mm}$ in vivo was achieved using OR-PAM at $586 \mathrm{~nm}$, whereas a lateral resolution of $84 \mu \mathrm{m}$ and penetration depth of $\sim 2-\mathrm{mm}$ in vivo was achieved using AR-PAM at $532 \mathrm{~nm}$. ( ) The Authors. Published by SPIE under a Creative Commons Attribution 4.0 Unported License. Distribution or reproduction of this work in whole or in part requires full attribution of the original publication, including its DOI. [DOI: 10.1117/1.JBO.24.8.086001]

Keywords: photoacoustic imaging; photoacoustic microscopy; optical resolution photoacoustic microscopy (PAM); acoustic resolution PAM; microelectro mechanical systems scanner.
\end{abstract}

Paper 180678R received Dec. 21, 2018; accepted for publication Jul. 15, 2019; published online Aug. 19, 2019.

\section{Introduction}

Photoacoustic microscopy (PAM) is a promising, high-resolution biomedical imaging technique based on the photoacoustic effect, which provides high sensitivity toward optical absorption at greater depths. ${ }^{1-4}$ In PAM, the absorption of light by endogenous contrast agents (hemoglobin, melanin, water, etc.) will induce temperature rise and in turn produce acoustic waves. The generated acoustic waves are detected using a wideband transducer to generate volumetric images. Compared to other optical imaging techniques, such as confocal microscopy, optical coherence tomography, and two-photon microscopy, ${ }^{5-7}$ PAM does not rely on backscattered photons, but the detection of low scattered acoustic waves makes the technique suitable for microscopic imaging at greater depth. Based on system designs to yield different resolutions and penetration depth, PAM can be classified into optical-resolution (OR) and acoustic-resolution (AR) PAM. OR-PAM uses tight optical focusing ${ }^{8,9}$ to achieve a high lateral resolution of $0.32 \mu \mathrm{m}$ and penetration depth of up to $1 \mathrm{~mm} .{ }^{10-12}$ AR-PAM employs tight acoustic focusing and weak optical focusing to achieve imaging depth of up to $3 \mathrm{~mm}$ (>optical mean free path of $1 \mathrm{~mm}$ ) with a lateral resolution of $45 \mu \mathrm{m}$ at $570 \mathrm{~nm} \cdot{ }^{13-15} \mathrm{In}$ order to achieve resolution similar to OR-PAM $(\leq 5 \mu \mathrm{m})$ acoustically, transducers with a center frequency $>400 \mathrm{MHz}$ are needed, which in turn reduces the penetration depth to $<100 \mu \mathrm{m}$.

Typically, OR-PAM is used to image superficial vasculatures and AR-PAM is used to image deeper vasculatures under the skin. Since the same optical and acoustic components can be used for both OR-PAM and AR-PAM, it would be of great

*Address all correspondence to Chulhong Kim, E-mail: chulhong@postech.edu; Malini Olivo, E-mail: malini_olivo@sbic.a-star.edu.sg advantage to combine them and co-register superficial optical resolution images and deep acoustic resolution images. The scalable resolution and penetration depth achievable using such a combined OR-AR system makes complete utilization of highresolution photoacoustic imaging (PAI) technology at a reduced cost. Even though it is challenging to integrate both OR- and AR-PAM systems together, several approaches have been taken to combine both OR and AR imaging modes in a single system. Simultaneous acquisition of OR- and AR-PAM images using raster scanning was reported previously using two lasers and a fiber bundle for light delivery. ${ }^{16}$ However, the acquisition times were long, due to the two-dimensional (2-D) mechanical scanning. In another approach, the acoustic and optical foci were shifted to achieve hybrid OR and AR imaging. ${ }^{17}$ Since the optical and acoustic foci are not confocally aligned, the resolution and image quality are not optimal. Switchable OR-ARPAM systems using a single-laser source was also reported. ${ }^{18,19}$ Since the system uses a single laser, after OR-PAM scanning the user needs to manually move the mechanical stage for translation to AR imaging. Even though the system was able to scan the same area, it was not simultaneous imaging. A miniaturized probe for achieving both optical and acoustic resolution was reported. ${ }^{20}$ However, the imaging speed was slow because the probe was attached to a 2-D motorized stage similar to previous approaches by other groups. The use of a single laser for achieving both OR- and AR-PAM images reduces the cost of the system but significantly increases the scan time and makes simultaneous imaging impossible. Moreover, raster scanning systems have larger scanning times and also not ideal for the development of miniaturized or handheld devices.

Imaging speed limitation in OR-PAM has improved significantly with the MEMS scanner. ${ }^{21,22}$ Using a MEMS scanner in the optical resolution mode (MEMS scanner-based OR-PAM), miniaturized handheld PAI devices have been developed. ${ }^{23,24}$ 
The shallow penetration depth of OR-PAM limits the use of such devices for a wide range of clinical applications, where imaging depth of $>1 \mathrm{~mm}$ will be needed. Here, we report an integrated high-speed OR-AR-PAM system for simultaneous acquisition of superficial high-resolution images and deep tissue images at lower resolution with a $50-\mu$ s delay between each acquisition. The simultaneous OR-AR-PAM imaging can be exploited for preclinical imaging applications including angiogenesis, drug response, biomarkers, brain functions, and gene activities, where acquiring high-resolution superficial images and low-resolution deeper tissue images will be beneficial. The combined OR-AR-PAM imaging using the MEMS scanner will be beneficial for the development of miniaturized handheld scanning devices particularly, for the detection of microvascular changes which occur as part of inflammatory skin disorders such as eczema and psoriasis. For clinicians to diagnose the severity and drug response for these skin conditions, imaging modalities for the visualization and quantification of high-resolution superficial structures, such as capillary loops and deep dermal vascular structures, are needed to offer complementary information about the underlying pathologies in the skin. When high-resolution structures like capillary loops cannot be resolved easily using AR-PAM and the vasculatures found deep in dermis cannot be resolved using OR-PAM, a combined OR-AR-PAM will be highly beneficial to get the best of both layers. High resolution in deep tissue is the ultimate goal of all biomedical imaging in the field. None of the present modalities show high resolution and deep imaging. Hence, the proposed OR-AR-PAM system will potentially fulfill the ultimate goal of high-resolution deep tissue imaging for various biomedical imaging applications.

The use of the MEMS scanner for PAI will help to improve the image acquisition time significantly compared to raster scanning systems, so that the image visualization is almost in real time. The ability to acquire both OR- and AR-PAM images using MEMS will help to visualize high-resolution superficial vasculatures as well as lower resolution deep vasculature, hence utilizing the full potential of high-resolution photoacoustic technology at high speed. Moreover, the use of MEMS will help in the development of miniaturized handheld based devices for clinical imaging applications.

\section{Methods}

\subsection{Simultaneous OR-AR-PAM System}

The schematic diagram of the high-speed OR-AR-PAM is shown in Fig. 1(a). The system employs two nanosecond laser systems operating at $532 \mathrm{~nm}$ (AWAVE-VIS-532, Advanced Optowave) and $586 \mathrm{~nm}$ (AWAVE-VIS-586, Advanced Optowave) operating at $5-\mathrm{kHz}$ repetition rate. For OR-PAM imaging, the beam from a 586-nm laser was passed through a telescope arrangement consisting of a double concave lens, i.e., L1 (LD2297, Thorlabs) and a plano-convex lens, L2 (LA1509, Thorlabs). The output power of the laser was controlled using a variable neutral density filter (NDF) (NDC-50C-2M, Thorlabs), before passing through an iris (ID12Z/M, Thorlabs). A fiber coupler (FC) (F240FC-532, Thorlabs) was used to couple the beam to a $10-\mu \mathrm{m}$ core multimode fiber (MMF) (M64L01, Thorlabs). An achromatic doublet, L3 (49-764, Edmund Optics), collimated the laser beam and another achromatic doublet, L4 (49-766, Edmund Optics) tightly focused the collimated beam.

For AR-PAM imaging, the beam from the 532-nm laser was attenuated and reshaped by a similar NDF and Iris. A similar FC as before was used to couple the beam to a $400-\mu \mathrm{m}$ core MMF (M74L01, Thorlabs). The output beam from the fiber was collimated using an objective lens (N4X-PF, Thorlabs) and deflected by a dichroic mirror (86334, Edmund Optics) to the same focusing achromatic doublet used for OR-PAM. The optical and acoustic focuses of the OR-AR-PAM system were aligned using an optical and acoustic beam combining system as described before. ${ }^{14}$ In short, a combination of an uncoated prism (NT32-330, Edmund Optics), and an aluminum-coated prism (NT32-331, Edmund Optics) provides optical reflection and acoustic transmission. On top of the combiner, a plano-convex lens (CL, NT67-147, Edmund Optics) was attached for optical aberration corrections. On the right of the combiner, an acoustic lens (AL, NT45-010, Edmund Optics) was placed, which provides acoustic focusing. A 50-MHz transducer (V214-BC-RM, Olympus) was attached to the left side of the combiner to detect the acoustic signals. A single-axis MEMS scanner (Opticho, Republic of Korea) was used for $X$ axis scanning.

The scanning range for the MEMS scanner was $2 \mathrm{~mm}$. For more than 2-mm scanning range, we used a MEMS scanner for $X$ axis and mechanical stage for $Y$ axis movement. A commercial photoacoustic system from Microphotoacoustics Inc. (New York) was used for $Y$ axis movement and signal acquisition. A 2-axis MEMS scanner can be used instead of a single-axis MEMS for small area imaging, which is ideal for the development of miniaturized handheld devices. ${ }^{23}$ The 532 and $586 \mathrm{~nm}$ wavelengths used for AR-PAM and OR-PAM imaging were near two particular isobestic absorption points of oxy- and deoxy-hemoglobin, i.e., at 530 and $584 \mathrm{~nm}$. For future applications involving the use of contrast agents, lasers having wide tunability range like dye laser or optical parametric oscillator will be needed.

The system can operate either in switchable or simultaneous imaging modes. In the switchable mode, the system can operate sequentially in either OR or AR mode of operation. For simultaneous imaging, two lasers were triggered alternately with a $50-\mu$ s delay to produce dual A-line (depth resolved 1-D profile) pairs [Fig. 1(b)]. The time delay between the laser triggers will help in the separation of PA signals acquired in a single A-line. The time delay between two laser pulses can be adjusted depending on the repetition rate of both lasers. In each B-scan (depth resolved 2-D cross section), 200 A-line pairs were correlated [Fig. 1(c)]. The receiver will be triggered alternately at $50-\mu$ s intervals to collect the A-lines from both the lasers. A $Z$ axis stage was used in the system to position the optical and acoustic focus. Since our animal/sample holder also can be adjusted along the $Z$ axis mechanically, the $Z$ step size of the mechanical stage is not important in our set up since it was only used for the purpose of alignment.

\subsection{Laser Safety}

The maximum pulse energy by a single-laser pulse (MPE $\mathrm{SLP}_{\mathrm{SP}}$ ) on the skin surface should not exceed $2 C_{A} 10^{-2} \mathrm{~J} / \mathrm{cm}^{2}$, where $C_{A}$ is the wavelength correction factor, which is unity for visible wavelength range (400 to $700 \mathrm{~nm}$ ). If a single point on the skin is exposed to more than $10 \mathrm{~s}$, the irradiance should not exceed $200 \mathrm{~mW} / \mathrm{cm}^{2}$. In the case of MEMS scanning, a single point on the skin will not be exposed for $10 \mathrm{~s}$; hence, the maximum permissible exposure is limited by $1.1 C_{A} t^{0.25} \mathrm{~mJ} / \mathrm{cm}^{2}$, where $t$ is the exposure duration in seconds.

For AR-PAM, at the ultrasound focus, the size of the optical beam was $2 \mathrm{~mm}$. With a pixel separation of $10 \mu \mathrm{m}, 200$ adjacent 


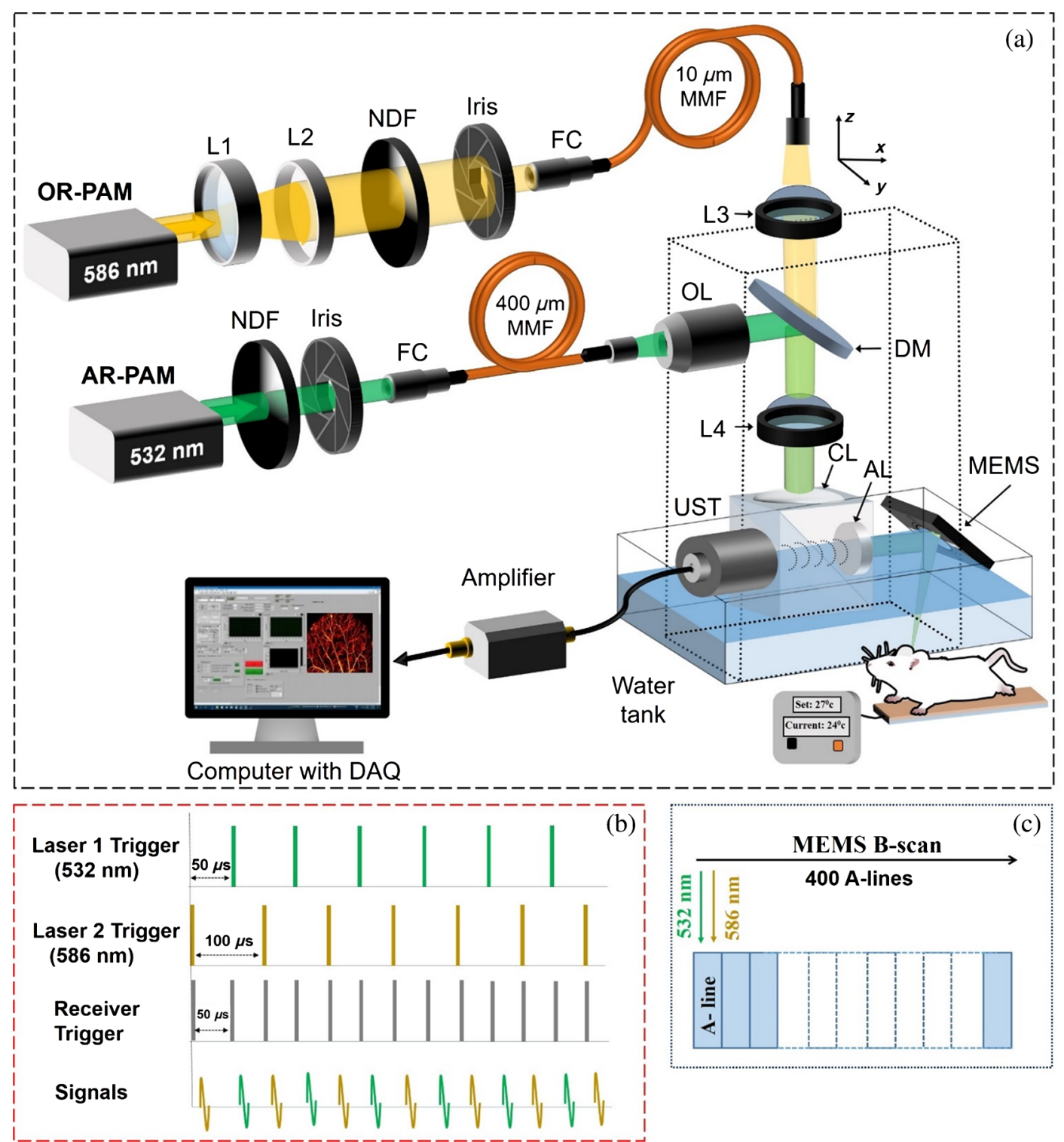

Fig. 1 (a) Schematic diagram of the OR-AR-PAM system. L1, concave lens; L2, convex lens; NDF, neutral density filter; FC, fiber coupler; UST, ultrasound transducer; OL, objective lens; MMF, multimode fiber; DAQ, data acquisition; $L 3$ and $L 4$, achromatic doublet; $C L$, correction lens; $A L$, acoustic lens. (b) Triggering sequence. (c) Data acquisition sequence in a single B-scan.

laser pulses overlap. At 5-kHz laser repetition, the exposure time was $0.04 \mathrm{~s}$, so the maximum pulse energy for the pulse train $\left(\mathrm{MPE}_{\text {TRAIN }}\right)$ was $490 \mathrm{~mJ} / \mathrm{cm}^{2}\left(1.1 C_{A} t^{0.25}\right)$. The AR-PAM system can deliver per pulse energy of $0.32 \mathrm{~mJ} / \mathrm{cm}^{2}(10 \mu \mathrm{J} /$ pulse, 2-mm-diameter focus) for mouse ear imaging and $0.95 \mathrm{~mJ} / \mathrm{cm}^{2}$ (30 $\mu \mathrm{J} /$ pulse, 2-mm-diameter focus) for dorsal and lower limb imaging, which is well below the MPE $\mathrm{SLP}_{\mathrm{S}}$ safety limit.

For OR-PAM, we believe the effect of collimated beam size and optical aberrations might have reduced the objective NA from 0.25 to 0.06 , which will give a spot size diameter of $5 \mu \mathrm{m}$. Assuming the optical focus is $200 \mu \mathrm{m}$ below the skin surface, the surface spot size was $30-\mu \mathrm{m}$ in diameter. Having a pixel separation of $10 \mu \mathrm{m}$, three adjacent laser pulses overlap on the skin surface. The current OR-PAM system can deliver an $\mathrm{MPE}_{\mathrm{SLP}}$ of $14 \mathrm{~mJ} / \mathrm{cm}^{2}(100 \mathrm{~nJ} /$ pulse, $30 \mu \mathrm{m}$ at the skin surface) for ear imaging. In the case of dorsal and lower limb imaging, the optical focus will be $400 \mu \mathrm{m}$ below the skin surface and the surface spot size was $60-\mu \mathrm{m}$ in diameter. Having a minimum pixel separation of $10 \mu \mathrm{m}$, six adjacent laser pulses overlap on the skin surface. The current OR-PAM system can deliver an $\mathrm{MPE}_{\mathrm{SLP}}$ of $17.7 \mathrm{~mJ} / \mathrm{cm}^{2}(500 \mathrm{~nJ} /$ pulse, the spot size was $60-\mu \mathrm{m}$ in diameter at the skin surface).

Hence, the combined maximum pulse energy of the train including both OR-PAM and AR-PAM pulses will be $106 \mathrm{~mJ} / \mathrm{cm}^{2}\left(200 \times 0.32+3 \times 14 \mathrm{~mJ} / \mathrm{cm}^{2}\right)$ for ear imaging and $296 \mathrm{~mJ} / \mathrm{cm}^{2}\left(200 \times 0.95+6 \times 17.7 \mathrm{~mJ} / \mathrm{cm}^{2}\right)$ for dorsal

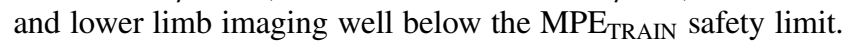

\section{Results and Discussion}

To characterize the OR-PAM lateral resolution, a test target having micrometer lines was scanned along the same edge with $0.5-\mu \mathrm{m}$ step size. The system was operated in the switchable mode to determine the resolution. Using edge spread function 

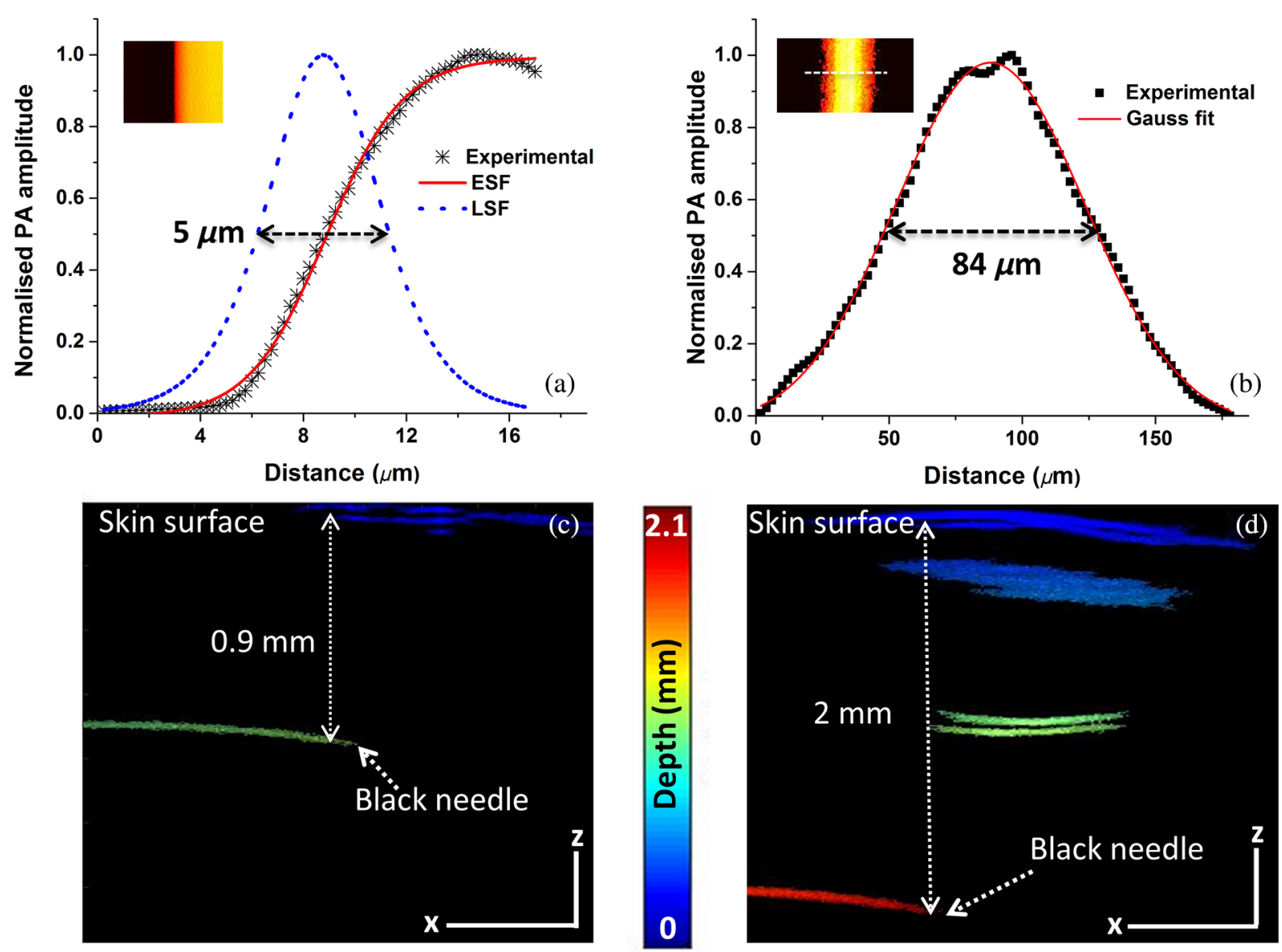

Fig. 2 Lateral resolution of the OR-AR-PAM system: (a) OR-PAM, black dots (*): photoacoustic signal; red line: ESF; blue line: LSF, inset shows MIP image of a resolution target. (b) AR-PAM, black dots ( $\mathbf{m})$ : photoacoustic signal; red line: Gaussian fit, inset shows MIP image of the carbon fiber. (c) B-scan ORPAM image of a black needle inserted into the mouse leg. (d) B-scan AR-PAM image of a black needle inserted into the mouse leg (scale bar: $500 \mu \mathrm{m}$ ).

(ESF), the data along the edge of the maximum intensity projection (MIP) image [inset in Fig. 2(a)] were fitted. The lateral resolution determined from the line-spread function (LSF) was $5 \mu \mathrm{m}$ for OR-PAM. Similarly, the lateral resolution of the ARPAM was characterized using carbon fiber with a size of $6 \mu \mathrm{m}$. The MIP image of the fiber [inset in Fig. 2(b)] was averaged and fitted to calculate the lateral resolution. The calculated lateral resolution from the full width at half maximum of the fitted data was $84 \mu \mathrm{m}$. The axial resolution of the system was $27 \mu \mathrm{m}$, which was determined from the axial profile of the carbon fiber.

To determine the maximum imaging depth of the system, a black needle inserted obliquely into the upper leg portion of a normal Balb/c mouse weighing $25 \mathrm{~g}$ was imaged. Figures 2(c) and 2(d) show the B-scan images of the needle acquired using OR- and AR-PAM. From the B-scan images, we could able to see the needle up to a depth of $0.9 \mathrm{~mm}$ below the skin surface using OR-PAM, whereas we were able to see the needle up to $2 \mathrm{~mm}$ from the skin surface using AR-PAM.

In the simultaneous OR-AR mode of imaging, two lasers were triggered alternately. The OR laser was operated at lower power and AR laser was operated at higher power. Using both the MEMS scanner and mechanical stages, the ear of a representative Balb/c nude mouse (25 to $27 \mathrm{~g}$ of weight) was imaged at $10-\mu \mathrm{m}$ step size. All guidelines by the Institutional Animal Care and Use Committee of SBIC, A*STAR, Singapore
(Protocol\#151085) were followed. A cocktail of ketamine $(120 \mathrm{mg} / \mathrm{kg})$ and xylazine $(16 \mathrm{mg} / \mathrm{kg})$ was used to anesthetize the animal. $0.1 \mathrm{ml} / 10 \mathrm{gm}$ of the mixture was injected intraperitoneally. Figure 3(a) shows the MIP images acquired using ORPAM, whereas Fig. 3(b) shows the MIP images acquired using AR-PAM of the mouse ear. The image acquisition time for an area of $7.5 \mathrm{~mm} \times 6 \mathrm{~mm}$ was $4 \mathrm{~min}$ at a laser repetition rate of $5 \mathrm{kHz}$. Figures 3(c) and 3(d) show the inset region of interest (ROI) area $(2.5 \mathrm{~mm} \times 4.5 \mathrm{~mm})$ scanned with a step size of $10 \mu \mathrm{m}$, which took $37 \mathrm{~s}$ to complete. Multiple $X-Y$ datasets were acquired and stitched together to obtain a large field of view. The delivered laser pulse energies during ear imaging for the OR-PAM and AR-PAM were $100 \mathrm{~nJ}$ and $10 \mu \mathrm{J}$, respectively.

In order to demonstrate the simultaneous imaging capability of the system at different imaging depths, a nude mouse was imaged at the abdomen dorsally. Figure 4 shows the PAM images acquired $(2 \mathrm{~mm} \times 3 \mathrm{~mm})$ using the OR-AR-PAM system. Figure 4(a) shows the photographed image of the dorsal side of the mouse abdomen with the black small box showing the imaging ROI. Figure 4(b) shows the OR-PAM MIP image, whereas Fig. 4(c) shows the AR-PAM MIP image. We could observe high-resolution superficial vasculature from the ORPAM image and deeper vasculatures from the AR-PAM image. Figures 4(d) and 4(e) show the depth encoded MIP images of the ROI shown in the OR- and AR-PAM images in Figs. 4(c) 

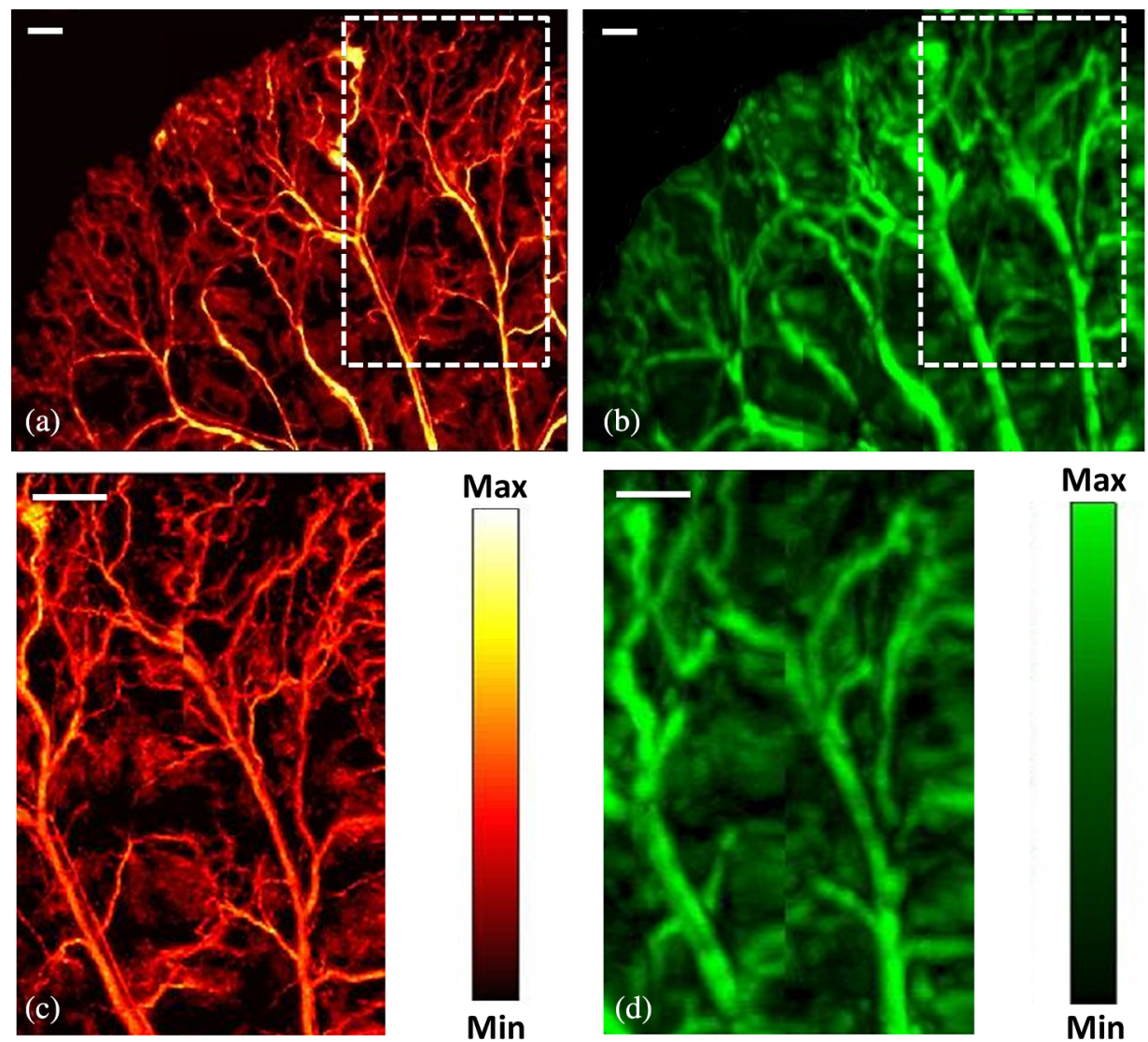

Fig. 3 MIP images of a mouse ear (a) OR-PAM, (b) AR-PAM, (c) ROI in OR-PAM, and (d) ROI in AR-PAM; scale bar: $500 \mu \mathrm{m}$.

and $4(\mathrm{~d})$, respectively. According to the previously reported article, $^{25}$ the OR-PAM resolution will degrade from $3 \mu \mathrm{m}$ to approximately $18 \mu \mathrm{m}$ at $0.9-\mathrm{mm}$ inside biological tissue. The maximum delivered laser pulse energies for the OR-PAM and AR-PAM were $500 \mathrm{~nJ}$ and $30 \mu \mathrm{J}$, respectively, well under the American National Standard Institute safety limit of $20 \mathrm{~mJ} / \mathrm{cm}^{2}$

Notably, the high-resolution vasculature structure cannot be distinguished using AR-PAM. Nevertheless, the acquired images from the OR-AR-PAM demonstrated the capability of the combined system to distinguish high-resolution superficial vasculatures from the low-resolution deep vasculatures in a single scan. Combining the effect of the MEMS scanner and mechanical stages, large areas can be imaged at high speed, making it suitable for imaging several biomedical applications.

There will be a mismatch between the simultaneously acquired B-scan signals using OR- and AR-PAM. The mismatch determined in our set up from the subsequent A-line signals was $66 \mu \mathrm{m}$. In order to achieve better 3-D co-registration accuracy, a co-registration algorithm with data correction will be needed. The motion artifacts are negligible when imaging mouse ear and minimal while imaging the dorsal side and the lower limb region. Motion artifacts could play a major role while imaging the abdominal regions of the mouse. Averaging multiple A-lines during data acquisition will reduce the effect of motion artifacts and incorporating motion correction algorithms ${ }^{26}$ will help in the better visualization of 3-D data. We will be developing such algorithms with motion correction or signal corrections for better co-registration and visualization in the future.
In previously reported studies, ${ }^{12,27}$ the time taken to complete OR-scan $(4.5 \mathrm{~mm} \times 5 \mathrm{~mm}$ ) area was $50 \mathrm{~min}$ (step size of $2 \mu \mathrm{m}$ in the $Y$ direction and $3 \mu \mathrm{m}$ in the $X$ direction). AR-PAM will take $8 \mathrm{~min}$ to complete same area (step size of $15 \mu \mathrm{m}$ in the $Y$ direction and $15 \mu \mathrm{m}$ in the $X$ direction). On the other hand, in this study, we used a MEMS scanner to simultaneously achieve OR-PAM and AR-PAM imaging. Using the MEMS scanner, a region of $2.5 \mathrm{~mm} \times 4.5 \mathrm{~mm}$ scanned using both OR-PAM and AR-PAM with a step size of $10 \mu \mathrm{m}$, took only $37 \mathrm{~s}$ to complete. To our knowledge, this is the first time, AR-PAM imaging and combined OR-AR-PAM imaging have been demonstrated using a MEMS scanner

In order to achieve optimum image quality for OR-PAM, the sampling step size should be half the lateral resolution. Since we are using a high-repetition rate laser for AR-PAM imaging, we have used a $10-\mu \mathrm{m}$ step size to reduce the AR-PAM imaging sampling rate. In an ideal set up in the future, we will design the AR-PAM laser with lower repetition rate and high energy and the triggering will be delayed accordingly so that after every 17 steps of OR-PAM signal acquisition the AR-PAM laser will be triggered to acquire the data.

In summary, we have demonstrated an integrated AR and OR-PAM system equipped with a MEMS scanner and mechanical stages for wide-area imaging at high speeds. This OR-ARPAM system can acquire shallow depth images at high-resolution and lower-resolution deep tissue imaging. The combined effect of the system was demonstrated by performing in vivo mouse ear and abdomen region imaging. A $5-\mu \mathrm{m}$ lateral resolution with $0.9-\mathrm{mm}$ imaging depth in vivo was achieved using 

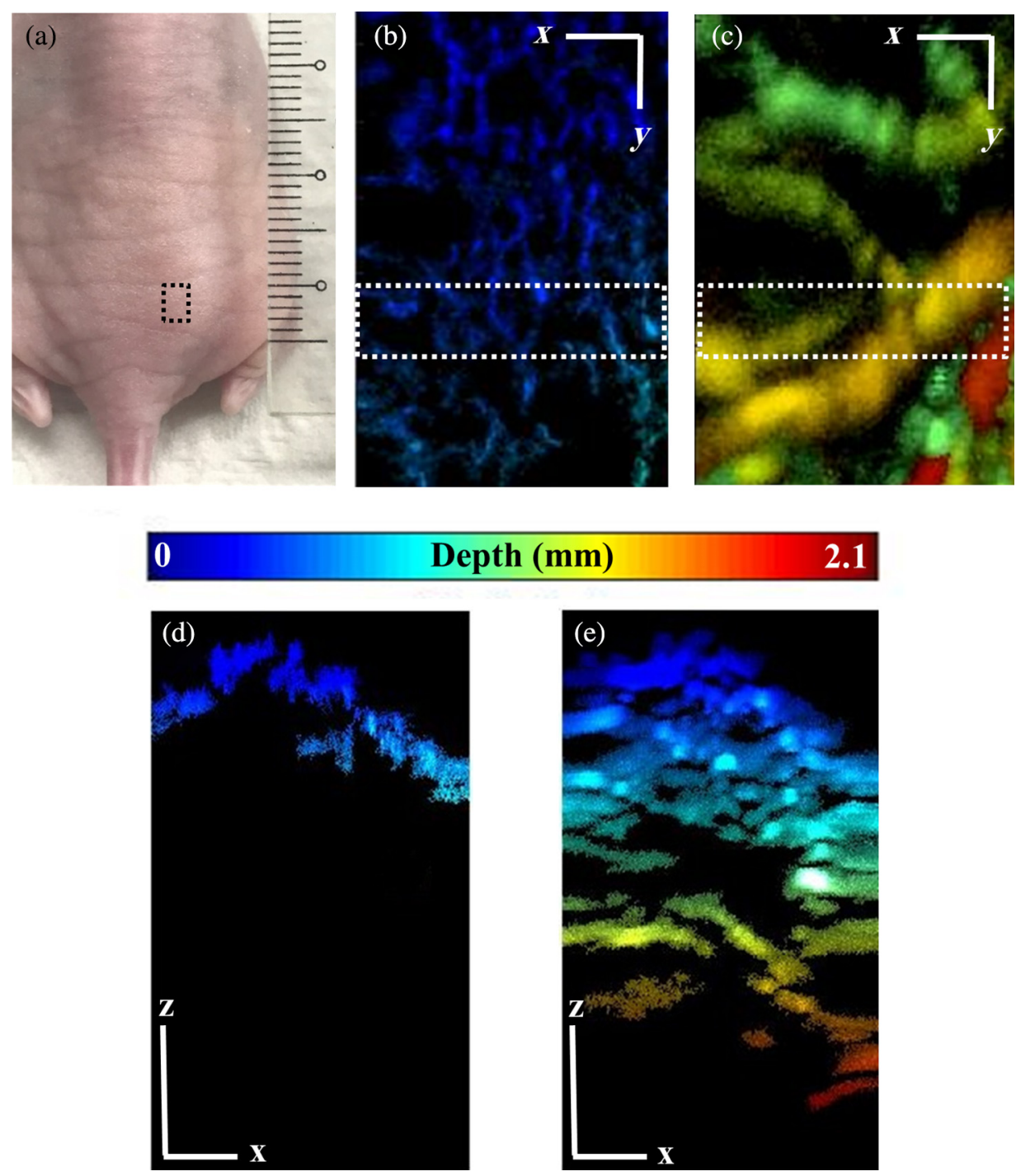

$\operatorname{Depth}(\mathbf{m m})$
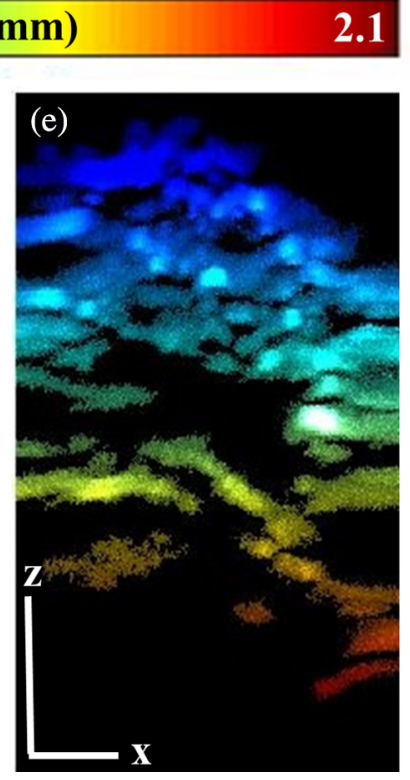

Fig. 4 MIP images of the dorsal side of the mouse abdomen: (a) photograph showing ROI in black box, (b) OR-PAM, (c) AR-PAM, (d) depth profile MIP of the ROI shown in OR-PAM image, and (e) depth profile MIP of the ROI shown in AR-PAM image (scale bar: $500 \mu \mathrm{m}$ ).

OR-PAM, whereas 84- $\mu \mathrm{m}$ lateral resolution with 2-mm imaging depth in vivo was achieved with AR-PAM. The simultaneous multiscale data acquisition capability at high speeds using both OR and AR modes makes the system highly suitable for various preclinical imaging applications. The combined OR-AR-PAM imaging using a MEMS scanner will be beneficial for the development of miniaturized handheld scanning devices, particularly for clinical skin imaging applications where visualization and quantification of high-resolution superficial structures such as capillary loops and deep dermal vascular structures will be needed. Moreover, the ability to achieve acoustic resolution images using a MEMS scanner makes the system compatible for plug-and-play switching to handheld probe mode for clinical translation. The use of higher pulse energies for the AR-PAM in this setup could significantly improve the imaging depth of ARPAM and efficiency of the proposed system.

\section{Conclusions}

To simultaneously utilize the benefits of optical and acoustic resolution modes in PAM at high speed, an integrated MEMS scanner-based OR-AR-PAM system was developed. The system can achieve high-resolution shallow depth images and low-resolution images at increased imaging depth. The system is capable of high-speed wide area scanning using MEMS and raster mechanical movement. Using a 50-MHz transducer, 5- $\mu$ m lateral resolution with 0.9-mm imaging depth in vivo was achieved using ORPAM and, 84- $\mu$ m lateral resolution with 2-mm imaging depth in vivo was achieved using AR-PAM. The axial resolution of the system was $27 \mu \mathrm{m}$. The combined effect of the system was demonstrated by performing in vivo imaging of mouse ear and abdomen region. The developed OR-AR-PAM system can be used for various preclinical imaging applications including angiogenesis, drug response, biomarkers, brain functions, and gene activities. Moreover, the combined OR-AR-PAM imaging achievable using the MEMS scanner will be beneficial for the clinical translation of miniaturized handheld scanning devices, mainly for skin imaging applications.

\section{Disclosures}

The authors declare that there are no conflicts of interest related to this article. C. Kim and J. Kim have financial interests in Opticho, which, however, did not support this work. 


\section{Acknowledgments}

This work was supported by intramural funding from Singapore Bioimaging Consortium, Biomedical Research Council of Agency for Science, Technology, and Research (A*STAR), Singapore. This research was also supported by grants from the Korea Health Technology R\&D Project (No. HI15C1817), through the Korea Health Industry Development Institute of the Ministry of Health and Welfare; the ICT Consilience Creative Program (No. IITP-2017-R0346-16-1007), through the Institute for Information and Communications Technology Promotion, Ministry of Science and ICT; and Pioneer Research Projects (No. 2017M3C1A3037762), through the National Research Foundation, Republic of Korea. The authors would also like to thank Ms. Hann Qian Lim, for help during in vivo experiments; Mr. Douglas Goh, for help in preparing the schematic of the imaging set-up; and Prof. Vincent Toal, Technological University Dublin, for proofreading the manuscript.

\section{References}

1. L. V. Wang and J. Yao, "A practical guide to photoacoustic tomography in the life sciences," Nat. Methods 13(8), 627-638 (2016).

2. J. Yao and L. V. Wang, "Photoacoustic brain imaging: from microscopic to macroscopic scales," Neurophotonics 1(1), 011003 (2014).

3. P. Beard, "Biomedical photoacoustic imaging," Interface Focus 1(4), 602-631 (2011).

4. R. Bi et al., "Photoacoustic microscopy for evaluating combretastatin A4 phosphate induced vascular disruption in orthotopic glioma," J. Biophotonics 11(10), e201700327 (2018).

5. R. M. Werkmeister et al., "Ultrahigh-resolution OCT imaging of the human cornea," Biomed. Opt. Express 8(2), 1221-1239 (2017).

6. S. Karpf et al., "Two-photon microscopy using fiber-based nanosecond excitation," Biomed. Opt. Express 7(7), 2432-2440 (2016).

7. Z. Wang et al., "In vivo vascular imaging with adaptive optics confocal scanning fluorescence microscopy," Proc. SPIE 9230, 92301W (2014).

8. S. Hu, K. Maslov, and L. V. Wang, "Second-generation optical-resolution photoacoustic microscopy with improved sensitivity and speed," Opt. Lett. 36(7), 1134-1136 (2011).

9. S. Jeon et al., "In vivo photoacoustic imaging of anterior ocular vasculature: a random sample consensus approach," Sci. Rep. 7(1), 4318 (2017).
10. W. Song et al., "Reflection-mode in vivo photoacoustic microscopy with subwavelength lateral resolution," Biomed. Opt. Express 5(12), 4235-4241 (2014).

11. M. Moothanchery et al., "Optical resolution photoacoustic microscopy based on multimode fibers," Biomed. Opt. Express 9(3), 1190-1197 (2018).

12. T. Chuangsuwanich et al., "Photoacoustic imaging of lamina cribrosa microcapillaries in porcine eyes," Appl. Opt. 57(17), 4865-4871 (2018).

13. S. Park et al., "Acoustic resolution photoacoustic microscopy," Biomed. Eng. Lett. 4(3), 213-222 (2014).

14. H. F. Zhang et al., "Functional photoacoustic microscopy for highresolution and noninvasive in vivo imaging," Nat. Biotechnol. 24(7), 848-851 (2006).

15. K. Maslov, G. Stoica, and L. V. Wang, "In vivo dark-field reflectionmode photoacoustic microscopy," Opt. Lett. 30(6), 625-627 (2005).

16. W. Xing et al., "Integrated optical-and acoustic-resolution photoacoustic microscopy based on an optical fiber bundle," Opt. Lett. 38(1), 52-54 (2013).

17. H. Estrada et al., "Real-time optoacoustic brain microscopy with hybrid optical and acoustic resolution," Laser Phys. Lett. 11(4), 045601 (2014).

18. M. Moothanchery, A. Sharma, and M. Pramanik, "Switchable acoustic and optical resolution photoacoustic microscopy for in vivo small-animal blood vasculature imaging," J. Visual. Exp. 124, e55810 (2017).

19. S. Jeon, J. Kim, and C. Kim, "In vivo switchable optical- and acousticresolution photoacoustic microscopy," Proc. SPIE 9708, 970845 (2016).

20. Z. Guo, Y. Li, and S.-L. Chen, "Miniature probe for in vivo opticaland acoustic-resolution photoacoustic microscopy," Opt. Lett. 43(5), 1119-1122 (2018).

21. J. Yao et al., "Wide-field fast-scanning photoacoustic microscopy based on a water-immersible MEMS scanning mirror," J. Biomed. Opt. 17(8), 080505 (2012).

22. J. Y. Kim et al., "Fast optical-resolution photoacoustic microscopy using a 2-axis water-proofing MEMS scanner," Sci. Rep. 5, 07932 (2015).

23. K. Park et al., "Handheld photoacoustic microscopy probe," Sci. Rep. 7(1), 13359 (2017).

24. L. Lin et al., "Handheld optical-resolution photoacoustic microscopy," J. Biomed. Opt. 22(4), 041002 (2017).

25. P. Hai et al., "Near-infrared optical-resolution photoacoustic microscopy," Opt. Lett. 39(17), 5192-5195 (2014).

26. M. Schwarz et al., "Motion correction in optoacoustic mesoscopy," Sci. Rep. 7, 10386 (2017).

27. M. Moothanchery and M. Pramanik, "Performance characterization of a switchable acoustic resolution and optical resolution photoacoustic microscopy system," Sensors (Basel) 17(2), 357 (2017).

Biographies of the authors are not available. 